\title{
Le système immunitaire à la naissance : entre l'apprentissage du soi et du non-soi
}

A la naissance, l'immaturité partielle des lymphocytes présents en périphérie se traduit par un déficit de production d'interleukines par les lymphocytes $T$ auxiliaires et un défaut de production d'immunoglobulines par les lymphocytes B. Contrairement aux adultes, les lymphocytes de nouveau-nés n'ont jamais été exposés à des antigènes étrangers. Cette différence pourrait rendre compte, au moins en partie, de l'immaturité de leur système immunitaire. En effet, les antigènes, viraux ou bactériens en particulier, sont responsables non seulement d'une activation transitoire des fonctions des lymphocytes périphériques mais également de la maturation vers un stade plus avancé de leur différenciation (lymphocytes mémoires), caractérisée par une plus grande efficacité fonctionnelle. C'est cette maturation lymphocytaire, associée à la présence d'anticorps spécifiques qui s'opposera à des infections ultérieures. L'immaturité des lymphocytes $\mathbf{T}$ du nouveau-né et leur maturation progressive se manifestent au niveau de l'expression de molécules de surface, appelées CD (cluster of differentiation), qui interviennent dans l'adhérence cellulaire et dans les processus d'activation.

Ghislaine Sterkers Hélène Pirenne-Ansart Assia Eljaafari-Corbin Yannick Aujard

\section{ADRESSES}

G. Sterkers : professeur des universités, praticien hospitalier, directeur du laboratoire. H. PirenneAnsart: chargée de recherche, non statutaire à l'Inserm. A. Eljaafari-Corbin : chargée de recherche, non statutaire à l'Inserm. Laboratoire du développement et de la maturation du système immunitaire. Y. Aujard : professeur des universités, praticien hos pitalier. Service de nćonatologie, hôpital Robert-Debré, 48, boulevard Sćrurier, 75019 Paris, Francc. $\mathrm{m} / \mathrm{s} n^{\circ} 3$ vol. 9 , mars 93 'immunité spécifique est assurée par les lymphocytes $\mathrm{T}$ et les lymphocytes B. La mise en action des lymphocytes est déclenchée, au décours de leur interaction avec les antigènes dont ils sont spécifiques, selon les modalités qui sont schématisées dans la figure 1. L'immunité spécifique concourt au maintien de l'intégrité de chaque individu en éliminant les substances qui lui sont étrangères. Pour être efficace sans être nuisible à l'hôte, ce système doit être capable de tolérer ses propres structures : le soi, et d'éliminer toute structure exprimant des antigènes étrangers : le non-soi. Une tolérance à l'égard du soi n'est pas génétiquement déterminée. Elle est le résultat de l'élimination ou de l'inactivation des cellules $\mathrm{T}$ et $\mathrm{B}$ autoréactives qui s'effectue, pour l'essentiel, pendant la vie fotale, période pendant laquelle le développement du système immunitaire s'effectue dans un environnement stérile.

La naissance constitue une période charnière à partir de laquelle des contaminations par des agents pathogènes deviennent possibles. Dans la période périnatale, les défenses immunitaires contre ce non-soi sont partiellement immatures. Cette situation rend compte d'une susceptibilité particulière du nouveau-né aux infections primitives, materno-fœtales et nosocomiales. Celles-ci sont aggravées par l'immaturité du compartiment non spécifique de l'immunité (Tableau I, p. 308).

Au cours de la petite enfance, les stimulations antigéniques itératives vont largement contribuer à la poursuite 


\section{RÉFÉRENCES}

1. Nikolic-Zugic J. Phenotypic and functional stages in the intrathymic development of $\alpha \beta T$ cells. Immunol Today $1991 ; 2$ : 12-5.

2. Fagard R, Danielian S. Rôle des tyrosine protéine kinases dans l'activation des lymphocytes T. médecine/sciences $1989 ; 5$ : 552-8.

3. Malissen M, Malissen B. Réarrangements somatiques des gènes du récepteur des lymphocytes T. médecine/sciences $1986 ; 2$ : 304-11

4. Lewin B. Generation of immune diversity involves reorganization of the genome. In : Lewin B, ed. Genes IV. New York Oxford University Press, 1990 : 705-29.

5. Grant SGN, O'Dell TJ, Karl KA, Stein PL, Soriano P, Kandel ER. Impaired longterm potentiation special learning and hippocampal development in Fyn mutant mice. Science 1992 ; 258 : 1903-10.

6. Rocha B, Penit C, Baron C, Vasseur F, Dautigny Nand Freitas A. Accumulation of bromodcoxyuridine labclled cells in central and peripheral lymphoid organs : minimal estimates of production and turnover rates of mature lymphocytes. Eur J Immunol $1990 ; 20$ : 1697-703.

7. Jones La, Kruisbeck AM. What is the mechanism of induction of intrathymic tolerance? Res Immunol 1992; 143 : 291-3.

8. Lo D. Tolerance to peripheral antigens must involve non-deletional mechanisms. Res Immunol $1992 ; 143$ : 296-9.

9. Kroemer G, Martincz-A C. Clonal deletion, anergy and immunosuppression are connected in series to guarantee selftolerance. Res Immunol 1992 ; 143 : 335-40.

10. Ehlers S, Smith KA. Differentiation of $\mathrm{T}$-cell lymphokinc gene expression : the in vitro acquisition of T-cell memory. $J$ Exp Med $1991 ; 173$ : 25-36.

11. Bryson YJ, Winter HS, Gard SE, Fischer TJ, Stiehm ER. Deficiency of immune interferon production by leukocytes of normal newborns. Cell Immunol $1980 ; 15$ : 191-200.

12. Papadogiannakis $\mathrm{N}$, Johnsen $\mathrm{SA}$, Olding LB. Monocyte regulated hyporesponsiveness of human cord blood lymphocytes to OKT3-monoclonal antibody induced mitogenesis. Scand J Immunol 1986; 23 : de la maturation du système immunitaire dans son ensemble. Cette maturation aura pour conséquences un accroissement des performances de la plupart des constituants du système immunitaire ainsi que le développement d'une mémoire immunitaire vis-à-vis de chaque micro-organisme que l'individu aura précédemment rencontré. Cette mémoire immunitaire permettra l'acquisition progressive d'une protection s'opposant à la réplication des pathogènes les plus fréquemment représentés dans l'environnement de chaque individu.

$\mathrm{Au}$ cours de leur ontogenèse, les lymphocytes $\mathrm{B}$ et $\mathrm{T}$ expriment, à leur surface et selon une chronologie précise, des molécules de surface appelées CD (cluster of differentiation). Ces molécules $\mathrm{CD}$ sont des récepteurs membranaires qui peuvent avoir pour ligands soit des facteurs de différenciation et de croissance, soit des pro- téines de la matrice extracellulaire, soit des protéines exprimées à la surface d'autres cellules. Les molécules CD participent activement à la transmission des signaux d'activation qui permettent aux lymphocytes mûrs d'acquérir les fonctions pour lesquelles ils sont programmés (figure 2). Il est probable que les molécules CD jouent également un rôle important dans l'ontogenèse et ce, dès leur apparition sur les précurseurs des lymphocytes. L'identification de leur rôle dans les processus de différenciation des lymphocytes immatures fait actuellement l'objet de nombreuses investigations.

\section{Molécules CD et ontogenèse des Iymphocytes $T$ [1]}

La chronologie d'apparition des molécules $\mathrm{CD}$, dont le rôle dans l'onto-
Tableau 1

FONCTIONS DES CONSTITUANTS DU SYSTĖME IMMUNITAIRE A LA NAISSANCE

\begin{tabular}{|c|c|c|}
\hline & Principal rôle connu & Fonction à la naissance \\
\hline \multicolumn{3}{|l|}{ Compartiment non spécifique } \\
\hline $\begin{array}{l}\text { Interférons non immuns l } \alpha \text { et } \\
\beta \text { ) } \\
\text { Cellules NK } \\
\text { Système du complément } \\
\text { Système phagocytaire }\end{array}$ & $\begin{array}{l}\text { s'opposent à la réplication des } \\
\text { virus dans les cellules hôtes } \\
\text { - tuent les cellules infectées } \\
\text { par des virus ou autres organis- } \\
\text { mes à réplication intracellulaire } \\
\text { - tuent les cellules tumorales } \\
\text { - Iyse des micro-organis- } \\
\text { mes } \\
\text { - favorise la phagocytose } \\
\text { - lyse des micro-organis- } \\
\text { mes après phagocytose } \\
\text { - présentation des antigènes } \\
\text { aux lymphocytes T (macropha- } \\
\text { ges) }\end{array}$ & $\begin{array}{l}\text { Normale } \\
\text { Diminuée } \\
\text { Diminuée } \\
\text { Diminuée } \\
\text { ND* }\end{array}$ \\
\hline \multicolumn{3}{|l|}{ Compartiment spécifique } \\
\hline $\begin{array}{l}\text { Lymphocytes B } \\
\text { Lymphocytes } \mathrm{T} \text { auxiliaires } \\
\text { (CD4) } \\
\text { Lymphocytes T cytotoxiques } \\
\text { (CD8) }\end{array}$ & $\begin{array}{l}\text { - produisent des anticorps } \\
\text { - présentent les antigènes aux } \\
\text { Iymphocytes T auxiliaires } \\
\text { - produisent des interleu- } \\
\text { kines } \\
\text { - aident les lymphocytes B à } \\
\text { produire des lg } \\
\text { - tuent les cellules infectées } \\
\text { par des virus ou des germes à } \\
\text { réplication intracellulaire }\end{array}$ & $\begin{array}{l}\text { Diminuée } \\
\text { ND* } \\
\text { Diminuée } \\
\text { Diminuée } \\
\text { ND* }\end{array}$ \\
\hline
\end{tabular}

ND : non ou peu documenté. 
genèse des lymphocytes $T$ fait actuellement l'objet d'études extensives, est indiquée dans la figure 3. La molécule CD2 est exprimée précocement. Son ligand est la molécule CD58 (LFA3) qui est présente sur toutes les cellules spécialisées dans la présentation d'antigènes aux cellules $T$ (figure 2) incluant les cellules présentatrices d'antigènes (CPAg) du thymus (cellules corticales thymiques et dendritiques thymiques en particulier). L'interaction CD2/CD58 stabilise l'interaction entre les cellules $\mathrm{T}$ et les
CPAg. La molécule CD2 peut également transduire des signaux d'activation dans la cellule. Ce rôle de CD2 n'a été observé qu'à partir du stade de différenciation des thymocytes communs indiquant que l'expression membranaire du récepteur $\mathrm{T}$ est nécessaire à cette fonction. Comme le montre la figure 3, les molécules CD4 et CD8 sont coexprimées à la surface des thymocytes corticaux. A un stade plus tardif de leur maturation, les thymocytes médullaires puis les lymphocytes $T$ mûrs auxiliaires ou cytotoxiques n'expriment que les molécules CD4 ou CD8. Ainsi, les molécules CD4 sont exprimées exclusivement sur les cellules $T$ mûres ayant une fonction auxiliaire alors que les molécules CD8 sont exprimées exclusivement sur les cellules $\mathrm{T}$ mûres ayant des fonctions cytotoxiques. La nonexpression de CD4 ou de CD8 chez la squris affecte respectivement l'ontogenc̀se des lymphocytes $\mathrm{T}$ ayant des fonctions auxiliaires ou cytotoxiques, ce qui permet d'attribuer un rôle à

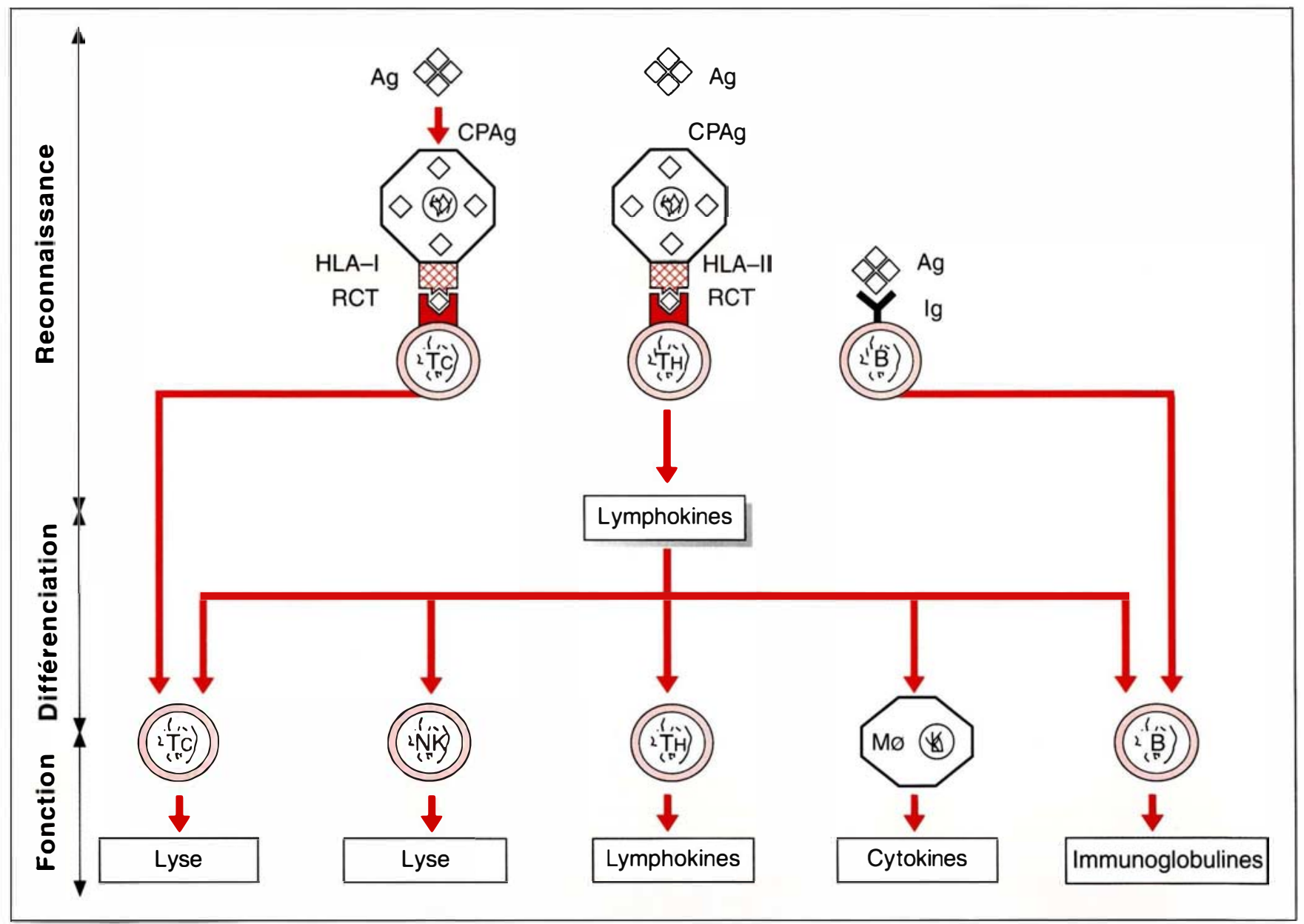

Figure 1. Schéma d'une réponse immunitaire spécifique. La mise en action des lymphocytes $B$ et $T$ est initiée par leur interaction avec les antigènes dont ils sont spécifiques. Cette reconnaissance se fait par l'intermédiaire des immunoglobulines membranaires des lymphocytes $B(\mathrm{lg})$ et du récepteur spécifique pour l'antigène des cellules $T$ (RcT). Contrairement aux lymphocytes $B(B)$, qui reconnaissent les antigènes sous leur forme native, les lymphocytes $T$ auxilliaires (T helper, $T h$ ) ou $T$ cytotoxiques $(T c)$ reconnaissent des fragments peptidiques d'antigènes fixés sur les molécules du complexe majeur d'histocompatibilité HLA [34]. Ces complexes HLA/peptides sont exprimés à la surface de cellules présentatrices d'antigène (CPAg). Les lymphocytes Th sont spécialisés dans la production de lymphokines. Les lymphokines sont des facteurs de différenciation et de croissance qui amplifient les fonctions des lymphocytes Th eux-mêmes. Ils contrôlent, par ailleurs, la mise en action des lymphocytes Tc et B qui appartiennent au compartiment de l'immunité spécifique. Enfin, ils amplifient les fonctions de constituants de l'immunité non spécifique tels que les cellules natural killer et les monocytes/macrophages.

$\mathrm{m} / \mathrm{s} n^{\circ} 3$ vol. 9, mars 93 


\section{RÉFÉRENCES}

13. Gerli R, Bertotto A, Crupi S, et al. Activation of cord T lymphocytes. I. Evidence for a defective $\mathrm{T}$-cell mitogenesis induced through the CD2 molccule. J. Immunol 1989; 142: 2853-9.

14. Lewis DB, Larsen A, Wilson CB. Reduced interferon-gamma RNA levels in human neonates : evidence for an intrinsic T-cell deficiency independent of other genes in T-cell activation. J Exp Med $1986 ; 163$ : 1018-23.

15. Sancho L, De La Hera A, Casas J, Vaquer S, Martinez C, Alvarcs-Mon M. Two different maturational stages of natural killer lymphocytes in human newborn infants. J Pediatr 1991; 119 : 446-54.

16. Hayward AR. Devclopment of lymphocyte responses and interactions in the human fetus and newborn. Immunol Rev $1981 ; 57: 39-59$.

17. Hayward A, Laszlo M, Vafai A Human newborn natural killer cell responses to activation by monoclonal antibodics, effect of culture with Herpes simplex virus. Immunol 1989 ; 142 : 1139-43.

18. Pirruccello SJ, Collins M, Wilson JE, McManus BM. Agc-rclated changes in naive and memory $\mathrm{CD} 4^{+} \mathrm{T}$ cells in healthy human children. Clin Immunol Immunopathol 1989 ; 52 : 341-5.

19. Mustclil T, Coggeshall KM, Altman A Rapid activation of the T-cell tyrosine protein kinase $\mathrm{p} 56^{\text {lck }}$ by the CD 45 phosphotyrosine phosphatase. Proc Natl Acad Sci USA 1989 ; $86: 6302-6$.

20. Cooke YT, Abraham KM, Forbush KA, Perlmutter RM. Regulation of T-cell receptor signaling by a SRC family protcin tyrosine kinase $\left(\mathrm{p} 59^{\mathrm{fyn}}\right)$. Cell 1991; 61: 281-91.

21. Mittler RS, Rankin BM, Kierner P. Physical associations between CD45 and CD4 or CD8 occur as late activation events in antigen receptor-stimulated human I cclls. J Immunol 1991; 147 : 3434-40.

22. Sanders ME, Markgoba MW, Sharrow SO, Stephany D, Springer 'TA, Young HA, Shaw S. Human memory $\mathrm{T}$ lymphocytes express increased levels of three cell adhe sion molecules (LFA-3, CD2 and LFA-1) and three other molccules (UCHL1, CD29 and Pgp-1), and have enhanced IFN gamma production. J Immunol $1988 ; 140: 1401-7$.

23. Sanders ME, Makgoba MW, Shaw S. Human naive and memory $T$ cells : reinterpretation of helper inducer and suppressor-inducer subscts. Immunol Today

ces molécules dans l'ontogenèse des thymocytes T. Les molécules CD4 ou CD8 sont couplées à une protéine tyrosine kinase de la famille Src : la p56 $6^{\text {lck }}$ [2], dont le rôle dans l'activation des lymphocytes $T$ mûrs a été clairement établi (figure 2). La création de souris mutantes n'exprimant pas la p56 lck $\left(\mathrm{m} / \mathrm{s} n^{\circ} 8\right.$, vol. 5, p. 589) entraîne des anomalies de différenciation des thymocytes. Cette observation suggère que cette enzyme est également impliquée dans l'ontogenèse thymique.
L'hypothèse de son intervention via CD4 et CD8 est renforcée par la possibilité d'activer la p56 $6^{\text {lck }}$ lors de la stimulation des molécules CD4 ou CD8 dans les cellules $\mathrm{T}$ à partir du stade de différenciation thymocyte commun.

L'apparition des molécules CD8 et CD4 précède l'expression membranaire du récepteur des cellules $T$ pour l'antigène $(\mathrm{RcT})$. Le RcT est la structure responsable de la reconnaissance spécifique de l'antigène (Ag) par les cellules $\mathrm{T}$ (figure 2). Il

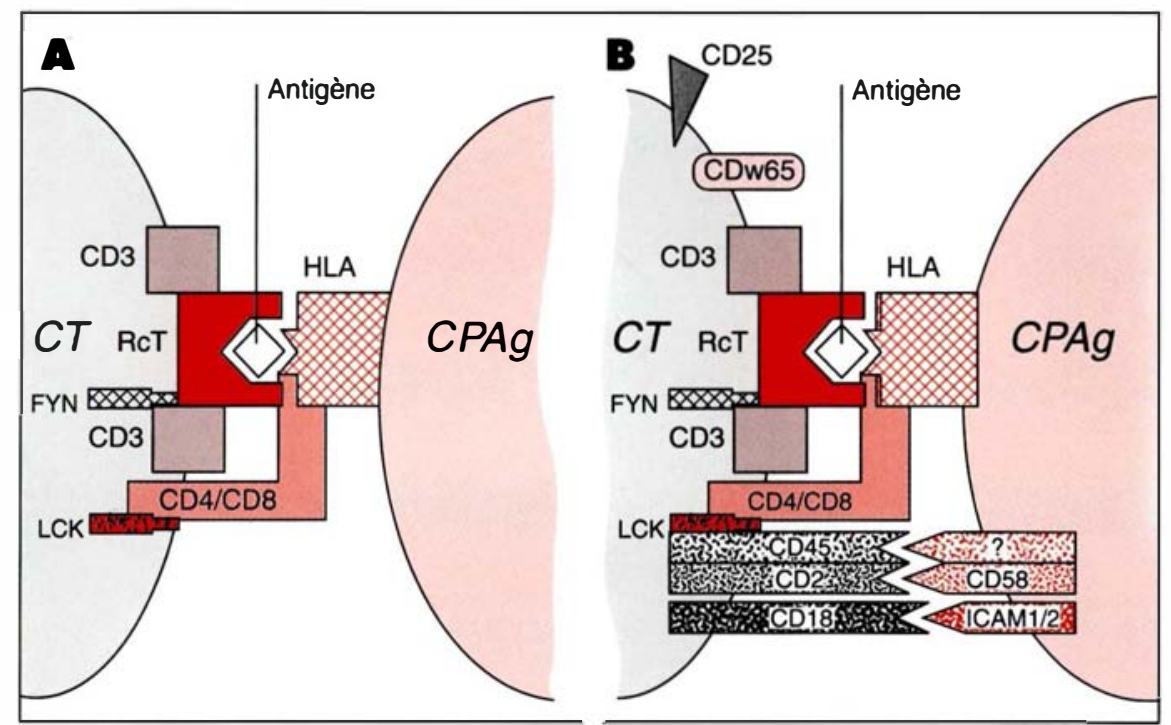

Figure 2. Reconnaissance de l'antigène par les cellules T. (A) La reconnaissance de l'antigène par les cellules $T$ se fait au cours d'une étroite coopération entre cellules $T$ et cellules présentatrices d'antigènes (CPAg). Elle met en jeu de multiples interactions moléculaires. Le récepteur pour l'Ag (RcT) interagit avec un fragment peptidique $d^{\prime} A g$ fixé dans une région hautement polymorphique des molécules HLA. Le RcT est associé au complexe polypeptidique CD3 qui assure la transduction de signaux d'activation à l'intérieur des cellules $T$. Les molécules $C D 4$ et $C D 8$, en interagissant avec une région non polymorphique de HLA, augmente l'affinité de l'interaction entre cellule $T$ et CPAg. (B) Un rôle dans l'adhérence intercellulaire a été attribué également à d'autres molécules exprimées à la surface des cellules $T$ telles que CD2 et CD 18 qui se lient respectivement à CD58 et ICAM 1 et 2 sur les CPAg. Des études plus récentes ont indiqué que ces molécules peuvent également modifier les signaux d'activation transmis par le RcT par l'intermédiaire de leur couplage à des enzymes impliquées dans la différenciation cellulaire. Tel est le cas de CD4/CD8 associés à une tyrosine kinase : la p56 ou le CD2 qui amplifie les signaux transmis par le RcT selon des modalités encore mal connues. Le ligand de certains $C D$ tel CD45 est inconnu. En revanche, le rôle de cette molécule, qui est une tyrosine phosphatase, dans la différenciation des lymphocytes fait actuellement l'objet de nombreuses études. Certaines molécules $C D$ ont pour ligand, non pas des molécules exprimées à la surface des CPAg mais des facteurs solubles tels que les interleukines ICD25, qui est le récepteur de l'interleukine 2, par exemple) ou des protéines de la matrice extracellulaire (CDw65, par exemple). L'expression des molécules $C D$ varie en fonction du stade de différenciation des cellules. L'identification de toutes les molécules $C D$ et la caractérisation précise de leur rôle lors des réponses immunitaires mais également au cours de l'ontogenèse restent un objectif à atteindre. FYN : tyrosine kinase p59Fyn. 


\begin{tabular}{|c|c|c|c|c|c|c|c|c|c|c|}
\hline \multirow[t]{2}{*}{$\mathrm{CD} 2$} & \multirow[t]{2}{*}{$\begin{array}{l}\mathrm{CD} 2 \\
\mathrm{CD} 8\end{array}$} & \multirow[t]{2}{*}{$\begin{array}{l}\text { CD2 } \\
\text { CD8 } \\
\text { RcT (c) }\end{array}$} & \multirow{2}{*}{$\begin{array}{l}\text { CD2 } \\
\text { CD8 } \\
\text { CD4 } \\
\text { RcT (c) }\end{array}$} & \multirow{2}{*}{$\begin{array}{l}\text { CD2 } \\
\text { CD8 } \\
\text { CD4 } \\
\text { CD5 } \\
\text { RcT (c) }\end{array}$} & \multirow{2}{*}{$\begin{array}{l}\text { CD2 } \\
\text { CD8 } \\
\text { CD4 } \\
\text { CD5 } \\
\text { RcT (c) }\end{array}$} & \multirow{2}{*}{$\begin{array}{l}\text { CD2 } \\
\text { CD8 } \\
\text { CD4 } \\
\text { CD5 } \\
\text { RcT (S) }\end{array}$} & \multirow{2}{*}{$\begin{array}{l}\text { CD2 } \\
\text { CD8 } \\
\text { CD5 } \\
\text { RCT (S) } \\
\text { CD45RA } \\
\text { CD2 } \\
\text { CD4 } \\
\text { CD5 } \\
\text { RCT (S) } \\
\text { CD45RA }\end{array}$} & $\begin{array}{l}\text { CD2 } \\
\text { CD8 } \\
\text { CD5 } \\
\text { RCT (S) } \\
\text { CD45RA }\end{array}$ & $\begin{array}{l}\text { CD2 } \\
\text { CD8 } \\
\text { CD5 } \\
\text { RcT (S) } \\
\text { CD45RO }\end{array}$ & $\begin{array}{l}\text { Cytotoxiques } \\
\text { suppresseurs }\end{array}$ \\
\hline & & & & & & & & $\begin{array}{l}\text { CD2 } \\
\text { CD4 } \\
\text { CD5 } \\
\text { RCT (S) } \\
\text { CD45RA }\end{array}$ & $\begin{array}{l}\text { CD2 } \\
\text { CD4 } \\
\text { CD5 } \\
\text { RcT (S) } \\
\text { CD45RO }\end{array}$ & Auxiliaires \\
\hline \multicolumn{3}{|c|}{ Thymocytes immatures } & \multicolumn{3}{|c|}{ Thymocytes communs } & & $\begin{array}{l}\text { Thymocytes } \\
\text { matures }\end{array}$ & \multicolumn{2}{|c|}{$\begin{array}{ll}\text { Lymphocytes } & \text { Lymphocytes } \\
\text { T matures } & \text { T matures } \\
\text { naîfs } & \text { mémoires }\end{array}$} & \\
\hline \multicolumn{7}{|c|}{ Corticale thymique } & $\begin{array}{l}\text { Médullaire } \\
\text { thymique }\end{array}$ & \multicolumn{2}{|c|}{$\begin{array}{l}\text { Organes lymphoïdes } \\
\text { périphériques }\end{array}$} & \\
\hline
\end{tabular}

Figure 3. Chronologie d'apparition des molécules CD au cours de l'ontogenèse des lymphocytes T. L'étude immunohistologique de sections de thymus de diverses espèces animales à différentes dates de gestation ainsi que des immunomanipulations permettant d'arrêter la maturation des thymocytes à différents stades de leur différenciation chez la souris ont largement contribué à définir la séquence d'apparition des molécules CD au cours de l'ontogenèse thymique. Dans un souci de simplification, la chronologie d'apparition des molécules CD les mieux connues est sélectivement indiquée dans cette figure. Des investigations complémentaires sont nécessaires pour définitivement extrapoler à l'homme le modèle proposé dans cette figure. A titre d'exemple, il n'y a pas actuellement de preuve chez I'homme de l'apparition des molécules CD8 avant celle des molécules CD4. (c) : présence intracytoplasmique; (S): présence à la surface des cellules.

est associé à CD3, un complexe polypeptidique composé d'au moins quatre molécules différentes. L'expression du RcT est l'aboutissement d'événements génétiques complexes et ordonnés de gènes [ 3 , 4] conférant à cette structure deux caractéristiques: (1) son extrême diversité (plus de $10^{15}$ RcT de séquences différentes peuvent être créés); (2) et sa clonalité (chaque thymocyte puis les lymphocytes $\mathrm{T}$ qui en dérivent expriment des RcT de séquence unique). CD3 est responsable de la transduction intracellulaire des signaux d'activation engendrés par l'interaction du RcT avec l'Ag. CD3 est couplé à une tyrosine kinase de la famille Src : p59 fyn (figure 2) dont le rôle dans l'ontogenèse thymique a été proposé (m/s $n^{\circ} 10$, vol. 8, p. 1091) [5].

\section{Développement du répertoire pré-immun des lymphocytes $T$ dans le thymus : acquisition d'une tolérance à l'égard du soi}

Il semble que la maturation des cellules $\mathrm{T}$ dans le thymus se fasse pré$\mathrm{m} / \mathrm{s} n^{\circ} 3$ vol. 9, mars 93 férentiellement pendant la vie fœtale. Les précurseurs des lymphocytes $\mathrm{T}$ sont formés initialement dans le foie fœtal puis dans la moelle osseuse. Ces précurseurs migrent de la moelle osseuse vers le thymus, organe dans lequel ils se différencient en prothymocytes puis en thymocytes et enfin en lymphocytes $T$ mûrs qui migrent dans les organes lymphoïdes périphériques. Après la naissance, la production de lymphocytes $\mathrm{T}$ issus du thymus se ralentirait. En effet, des études pratiquées chez la souris suggèrent que les mécanismes homéostatiques réglant la taille du pool des lymphocytes $\mathrm{T}$ à l'âge adulte privilégient un auto-renouvellement dans les organes lymphoïdes périphériques, plutôt qu'une génération de novo à partir des précurseurs hématopoïétiques [6].

Dès lors que les thymocytes expriment des RcT, la poursuite de leur maturation dans le thymus est conditionnée par la spécificité de ce récepteur (figure 4) (revue dans [7]). L'engagement des RcT dans une liaison de faible affinité avec les molécules du complexe majeur d'histocompatibilité (CMH : HLA chez l'homme) dont chaque individu a hérité et qui sont exprimées sur les cellules épithéliales thymiques est un prérequis à la poursuite de la maturation des thymocytes (sélection positive). Il est généralement admis que la " raison d'être " de cette sélection positive est de favoriser le développement des seules cellules $T$ efficaces car capables de reconnaître ultérieurement dans les organes lymphoïdes périphériques des antigènes étrangers présentés par des molécules du $\mathrm{CMH}$ autologues. Cette sélection positive est suivie d'une délétion (sélection négative) ( $\mathrm{m} / \mathrm{s} n^{\circ} 10$, vol. 4, p. $656 \mathrm{et}$ $n^{\circ} 10$, vol. 5, p. 788) ou d'une inactivation (anergie) $\left(\mathrm{m} / \mathrm{s} n^{\circ} 2\right.$, vol. 6 , p. 164) des cellules $\mathrm{T}$ exprimant des RcT de forte affinité pour les molécules du CMH autologues associées à des auto-Ag. La " raison d'être " de cette sélection négative est d'éliminer les cellules $\mathrm{T}$ potentiellement dangereuses car susceptibles d'induire des réactions auto-immunes après leur émergence du thymus. Ces processus de différenciation, proposés par Bevan en 1977, ont été récemment très élégamment démontrés grâce au développement de la technologie des souris trangéniques [8,9]. Dans ces modèles expérimentaux, le transgène 


\section{RÉFÉRENCES}

24. Clement LT, Yamashita N, Martin AM. The functionally distinct subpopulations of human $\mathrm{CD}^{+}{ }^{+}$helper/inducer $\mathrm{T}$ lymphocytes defined by anti-CD45 $R$ antibodies derive sequentially from a differentiation pathway that is regulated by activation-dependent post thymic differenciation. J Immunol 1988; 141: 1464-70.

25. Mackay CR. T-cell memory : the connection between function, phenotype and migration pathways. Immunol Today 1991 : $6: 189-91$

26. Pirenne H, Aujard Y, Eljaafari A, Bourillon A, Oury JF, Le Gac S, Blot P, Sterkers G. Comparison of $\mathrm{T}$-cell functional changes during childhood with the ontogeny of CDw29 and CD45RA expression on $\mathrm{CD}^{+}{ }^{+} \mathrm{T}$ ccll. Pediatr Res 1992 ; 32 : 81-6.

27. Mansar T, Wysocki LJ, Gridley T, Near RJ, Gefter ML. The molecular evolution of the immune response. Immunol Today $1985 ; 6$ : 94-101

28. Oslon JC, Leslie GA. Inheritance patterns of idiotype expression : maternal fetal immune regulatory network. Immunogenetics $1981 ; 13: 39-56$.

29. Baneck C, Milstein D. Mutation drift and repertoire shift in the maturation of the immune response. Immunol Rev 1987; 96 : 23-33.

30. Mansar T, Wysocki LJ, Margolies MN, Gefter ML. Evolution of antibody variable region during the immune response. Immunol Rev 1987; $96: 155-72$.

31. Magny JF. Intérêt des immunoglobulines intraveineuses pour la prophylaxie et le traitement des infections nćonatales. Arch $\mathrm{Fr}$ Pediatr 1992 ; 49 : 547-50.

32. Weisman LE, Stoll B, Kuester TJ, et al. Intravenous immune globulin therapy for carly onset sepsis in premature neonates. $J$ Pediatr $1992 ; 121$ : 434-43.

33. Cairo MS, Worcester CC, Rucker RW, Hanten S, Amlic RN, Sender L, Hicks DA. Randomized trial of granulocyte transfusions versus intravenous immun globulin therapy for neonatal neutropenia and sepsis. $J$ Pediatr $1992 ; 120: 281-5$

34. Rabourdin-Combe C, Bertolino $P$, Galin-Laurens V, Gerbier D. La présentation de l'antigène aux lymphocytes $\mathrm{T}$. médecine/sciences 1991; 7 : 674-80. d'un récepteur $\mathrm{T}$ de spécificité connue est exprimé à la surface de la plupart des thymocytes immatures. Chez des souris exprimant des molécules du CMH dont ce RcT est spécifique, les thymocytes exprimant les transgènes poursuivent leur maturation (sélection positive). En revanchc, ces thymocytes sont éliminés dans les thymus des souris exprimant des molécules du $\mathrm{CMH}$ associées à l'Ag dont ces RcT sont spécifiques (sélection négative). Il est vraisemblable que les cellules épithéliales thymiques serviraient de CPAg lors de la sélection positive tandis que les cellules dendritiques thymiques assureraient une sélection négative ([7], $\mathrm{m} / \mathrm{s} n^{\circ} 10$, vol. 4, p. 656 et $n^{\circ} 10$, vol. 5, p. 788). Les bases moléculaires de ces phénomènes de sélection positive et négative sont pratiquement inconnues. Les phénomènes de sélection positive et négative s'accompagnent d'une augmentation de la densité des RcT à la surface des thymocytes $\mathrm{CD}^{+} / \mathrm{CD} 8{ }^{+}$et de la disparition des molécules CD4 ou CD8 [1]. Les cellules $\mathrm{CD} 4+/ \mathrm{CD} 8-/ \mathrm{TcR}+/ \mathrm{CD} 3+$ ou $\mathrm{CD} 8+/ \mathrm{CD} 4-/ \mathrm{TcR}+/ \mathrm{CD} 3+$ qui cn résultent correspondent aux lymphocytes $\mathrm{T}$ mûrs auxiliaires ct cytotoxiques respectivement. Ces lymphocytes sont appelés pré-immuns car ils n'ont pas encore été en contact avec l'antigène dont ils sont spécifiques. Ils migrent vers les organes lymphoïdes périphériques où ils pourront acquérir respectivement des fonctions auxiliaires ou cytotoxiques/suppressives à la suite de leur interaction avec des antigènes étrangers.

\section{Particularités fonctionnelles et phénotypiques des lymphocytes T à la naissance}

L'acquisition d'une tolérance par des mécanismes de délétion ou d'inactivation de clones lymphocytaires semble se poursuivre pendant la période périnatale chez la souris. Chez l'homme, cette tolérance serait acquise avant la naissance.

A la naissance, les particularités fonctionnelles des lymphocytes $\mathrm{T}$ auxiliaires exprimant le marqueur CD4 ont fait l'objet, chez l'homme et dans diverses espèces animales, d'une particulière attention. La principale fonction de cette population est de produire des facteurs de différenciation et de croissance appelés cytokines (ou encore lymphokines ou interleukines) (figure 1). Les cytokines produites par les lymphocytes $\mathrm{CD}_{4}{ }^{+}$sont en particulier les interleukines 2, 3 et 4 , et l'interféron $\gamma$. Elles ont pour cibles toutes les cellules immunocompétentes. Par l'intermédiaire des cytokines qu'ils produisent, les lymphocytes auxiliaires $\mathrm{CD}^{+}{ }^{+}$favorisent la sécrétion des immunoglobulines par les lymphocytes B, assurent la différenciation des lymphocytes $\mathrm{T}$ $\mathrm{CD}^{+}$, cytotoxiques, et contrôlent en partie l'activation des macrophages et des cellules tueuses natural killer (NK). Les lymphocytes $\mathrm{T} \mathrm{CD}^{+}$isolés du sang de cordon ont un déficit de production des interleukines 3 et 4 , et de l'interféron $\gamma[10,11]$. Un déficit plus relatif de production d'interleukine 2 semble également exister [12, 13]. Ce défaut de production de lymphokines se situc en amont de la traduction puisque lc nivcau d'expression des ARN messagers correspondant $[10,14]$ est plus faible, voire indétectable, dans les lymphocytes $\mathrm{T}$ activés de sang de nouveau-né alors qu'il est élevé dans les lymphocytes $\mathrm{T}$ activés d'adultes. Les anomalies de production d'interleukines par les lymphocytes $\mathrm{T}$ auxiliaires à la naissance pourraient rendre compte (figure 1), au moins en partie, de l'incompétence relative des cellules NK [15], des lymphocytes B [16] et des lymphocytes $T$ cytotoxiques du nouveau-né (résultats non publiés). En effet, l'activité fonctionnelle de cellules NK et des lymphocytes cytotoxiques est sous le contrôle de l'IL-2 et de l'IFN $\gamma$. La production d'immunoglobulines (IgG) par les lymphocytes $\mathrm{B}$ dépend de la production d'IL-4, 5 et 6 par les lymphocytes $\mathrm{T}$ auxiliaires. Un déficit fonctionnel des lymphocytes B [16] et des cellules NK [17] et secondaires à leur propre immaturité semble également exister à la naissance.

Certaines des bases moléculaires responsables du déficit fonctionnel des lymphocytes $T$ à la naissance commencent à être explorées. En effet, les lymphocytes $T$ naïfs se distinguent des lymphocytes $T$ immuns, entre 
autres par une expression différentielle de certaines molécules $\mathrm{CD}$ à leur surface. Ainsi, contrairement à la majorité des lymphocytes $\mathrm{T}$ de l'adulte, qui expriment l'isoforme CD45RO, la majorité des lymphocytes $\mathrm{T}$ sanguins circulants chez le nouveau-né expriment l'isoforme CD45RA de la molécule membranaire CD45 [18]. Le rôle biologique de cette molécule CD45 a fait l'objet de nombreuses études au cours des cinq dernières années. Il s'agit d'une phosphatase qui, en déphosphorylant des tyrosine kinases, contrôle l'activité de celles-ci (figure 2) [19]. Or les formes activées de ces tyrosine kinases semblent jouer un rôle déterminant dans l'activation des lymphocytes $\mathrm{T}[20]$. Cette observation conduit naturellement à s'interroger sur les conséquences fonctionnelles de l'expression préférentielle de l'isoforme CD45RA à la surface des lymphocytes $\mathrm{T}$ des nouveau-nés. Par ailleurs, le RcT, les molécules CD4 et CD45 sont physiquement associés à la surface des lymphocytes immuns activés alors qu'ils ne le sont pas à la surface des lymphocytes préimmuns activés [21]. Cette dissociation pourrait être préjudiciable à l'efficacité de la transduction de signaux d'activation par le RcT lors de son interaction avec un antigène. Les lymphocytes $\mathrm{T}$ isolés du sang de cordon se caractérisent également par une faible densité d'expression d'autres molécules CD dont CD2 et CD18 [22]. Le rôle biologique connu de ces molécules est de favoriser la coopération entre lymphocytes $\mathrm{T}$ et cellules présentatrices d'antigène (figure 2). Ces molécules CD contribuent également à améliorer l'efficacité de la transduction de signaux d'activation via le RcT par des mécanismes plus ou moins bien caractérisés. Ainsi, il est probable que des anomalies quantitatives d'expression de ces molécules pourraient rendre compte, au moins en partie, des difficultés que les lymphocytes $\mathrm{T}$ du nouveau-né rencontrent pour acquérir, lorsqu'ils sont stimulés par l'antigène, les fonctions pour lesquelles ils sont programmés.

L'existence chez le nouveau-né d'unc activité accrue des lymphocytes $\mathrm{T}$ suppresseurs a été évoquée. En raison de la discordance des résultats rapportés dans la littérature dans ce domaine ainsi que des conceptions actuellement mouvantes de ces phénomènes de suppression [23], cet aspect ne sera pas discuté ici.

\section{Acquisition d'un répertoire $T$ immun après la naissance : adaptation à l'environnement}

L'interaction des lymphocytes $\mathrm{T}$ avec les antigènes étrangers dont ils sont spécifiques s'effectue dans les organes lymphoïdes périphériques (figure 5). Cette interaction est à l'origine d'événements biochimiques et génétiques responsables de l'acquisition transitoire par les lymphocytes ainsi stimulés, des fonctions $\mathrm{T}$ auxiliaires ou cytotoxiques pour lesquelles ils sont programmés. L'activation des lymphocytes T par l'Ag s'accompagne également de changements quantitatifs et qualitatifs durables. En

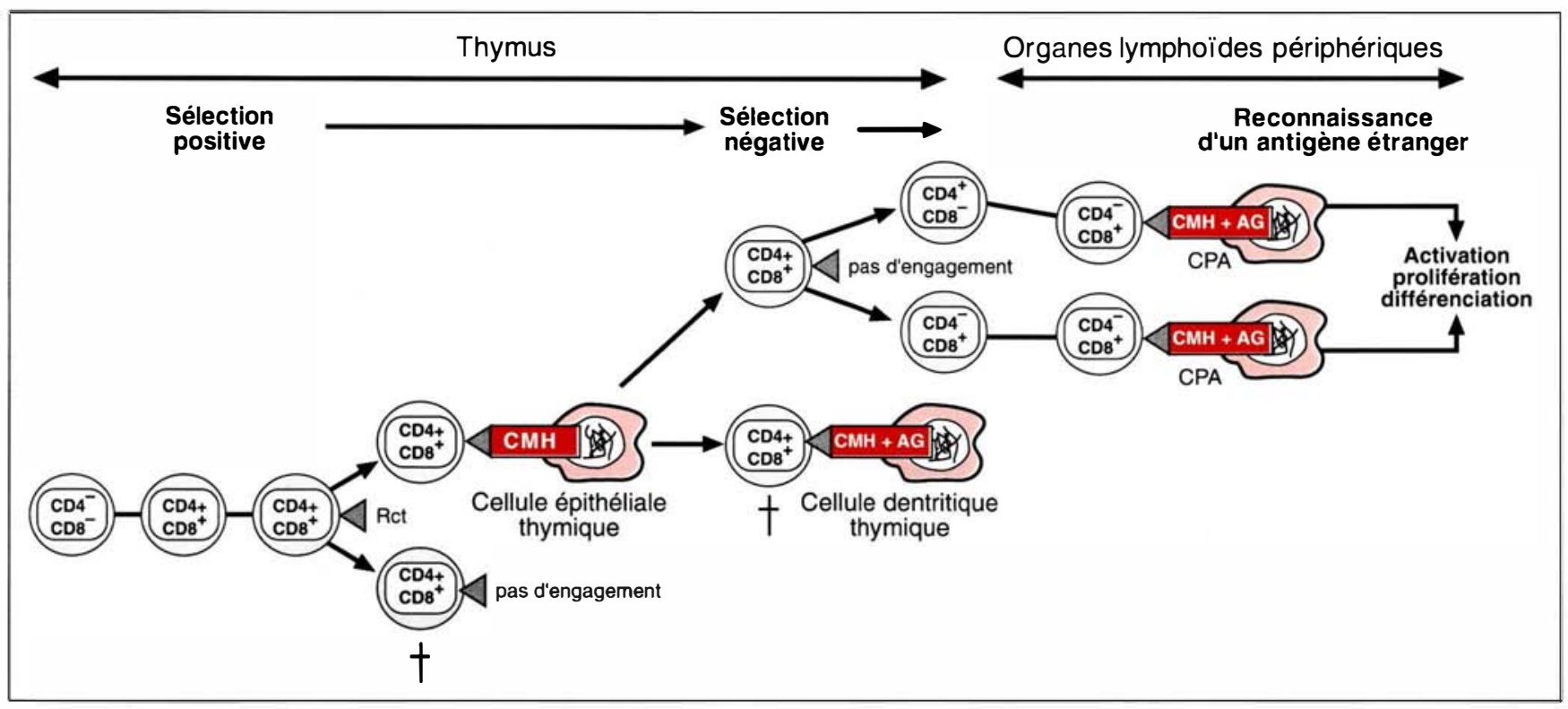

Figure 4. Sélection positive et négative dans le thymus. Le récepteur pour l'antigène (TcR) apparaît sur les thymocytes dans la corticale thymique. Son interaction avec les molécules du complexe majeur d'histocompatibilité sur les cellules épithéliales thymiques est un prérequis pour la poursuite de la maturation des thymocytes à ce stade de différenciation (sélection positive). Dans la médullaire thymique l'interaction des TcR avec des autoantigènes associés aux molécules du CMH entraîne la mort par apoptose des thymocytes autoréactifs (sélection négative). Au décours de ces deux événements de sélection, les cellules $T$ devenues des lymphocytes $T$ mûrs, migrent dans les organes lymphoides périphériques. Dans ces organes, l'interaction des TCR dans des liaisons de forte affinité avec des antigènes étrangers associés aux molécules du CMH permet aux lymphocytes $T$ auxiliaires $\mathrm{CD}^{+}$et $\mathrm{T}$ cytotoxique/suppresseur $\mathrm{CD} 8^{+}$ $d^{\prime}$ acquérir les fonctions pour lesquelles ils sont programmés. 
effet, les lymphocytes $\mathrm{T}$ stimulés par des antigènes subissent, d'une part, une expansion clonale et vont, d'autre part, progresser vers un stade plus avancé de leur différenciation. Cette maturation correspond au passage du stade de lymphocytes préimmuns (naïfs) à celui de lymphocytes immuns (mémoires). Ces phénomènes se traduisent par une augmentation d'expression de l'isof orme CD45RO de la molécule CD45 ainsi que par une augmentation d'expression des molécules d'adhésion et activation $\mathrm{CD} 2$ et CD18. Ces changements phénotypiques participent probablement à l'acquisition d'une plus grande efficacité fonctionnelle des lymphocytes "mémoires" compte tenu du rôle connu de ces CD [10, 24, 25].

Au cours de la petite enfance, les stimulations antigéniques itératives conduisent ainsi à l'accumulation progressive de lymphocytes $\mathrm{T}$ immuns particulièrement efficaces. Ces processus améliorent l'efficacité du système immunitairc dans son ensemble [26] et permettent l'acquisition progressive, au fil des années, d'une protcction contre les pathogènes les plus représentés dans le milieu dans lequcl l'enfant évolue.

\section{Ontogenèse des lymphocytes B (figure 5)}

De même que les lymphocytes $T$, les lymphocytes B dérivent d'un progéniteur hépatique (foie fœtal) puis médullaire. La différenciation de ces progéniteurs en lymphocytes B préimmuns s'effectue dans la moelle. Les différents stades de différenciation de la lignée $B$ se caractérisent par l'expression séquentielle de différentes molécules CD. Il a pu être démontré que certaines de ces structures étaient directement impliquées dans le processus de différenciation. Au stade de lymphocytes pré-B, des phénomènes de réarrangement des gènes qui codent pour les immunoglobulines (Ig) vont permettrc l'expression d'IgM à la surface de ces cellules. Les IgM membranaires constituent la structure de reconnaissance des lymphocytes B. Elle partage avec la structure de reconnaissance des lymphocytes $\mathrm{T}$ (RcT) deux caracté- seul type de récepteur par lymphocyte B) et son extrême diversité [27]. Contrairement aux RcT, les Ig interagissent spécifiquement avec des antigènes complexes en l'absence d'intervention des molécules HLA. Les mécanismes concourant au développement du répertoire $B$ pré-immun dans la moelle sont mal connus. Des phénomènes de délétion clonale semblent exister. Par ailleurs, le répertoire des cellules $\mathrm{T}$ influence celui des cellules B et réciproquement. Les anticorps eux-mêmes semblent inter- venir dans la régulation de l'acquisition du répertoire $B$. Au cours de la vie fœtale, des Ig d'origine maternelle traversent la barrière placentaire et peuvent ainsi influencer le répertoire de l'enfant [28]. La majorité du répertoire pré-immun semble acquis à la naissance. A la suite de leur maturation dans la moelle osseuse, les lymphocytes B pré-immuns migrent dans les organes lymphoïdes périphériques. Dans la période néonatale, de faibles quantités d'Ig, quasi exclusivement d'isotype $\operatorname{Ig} M$, sont produi-

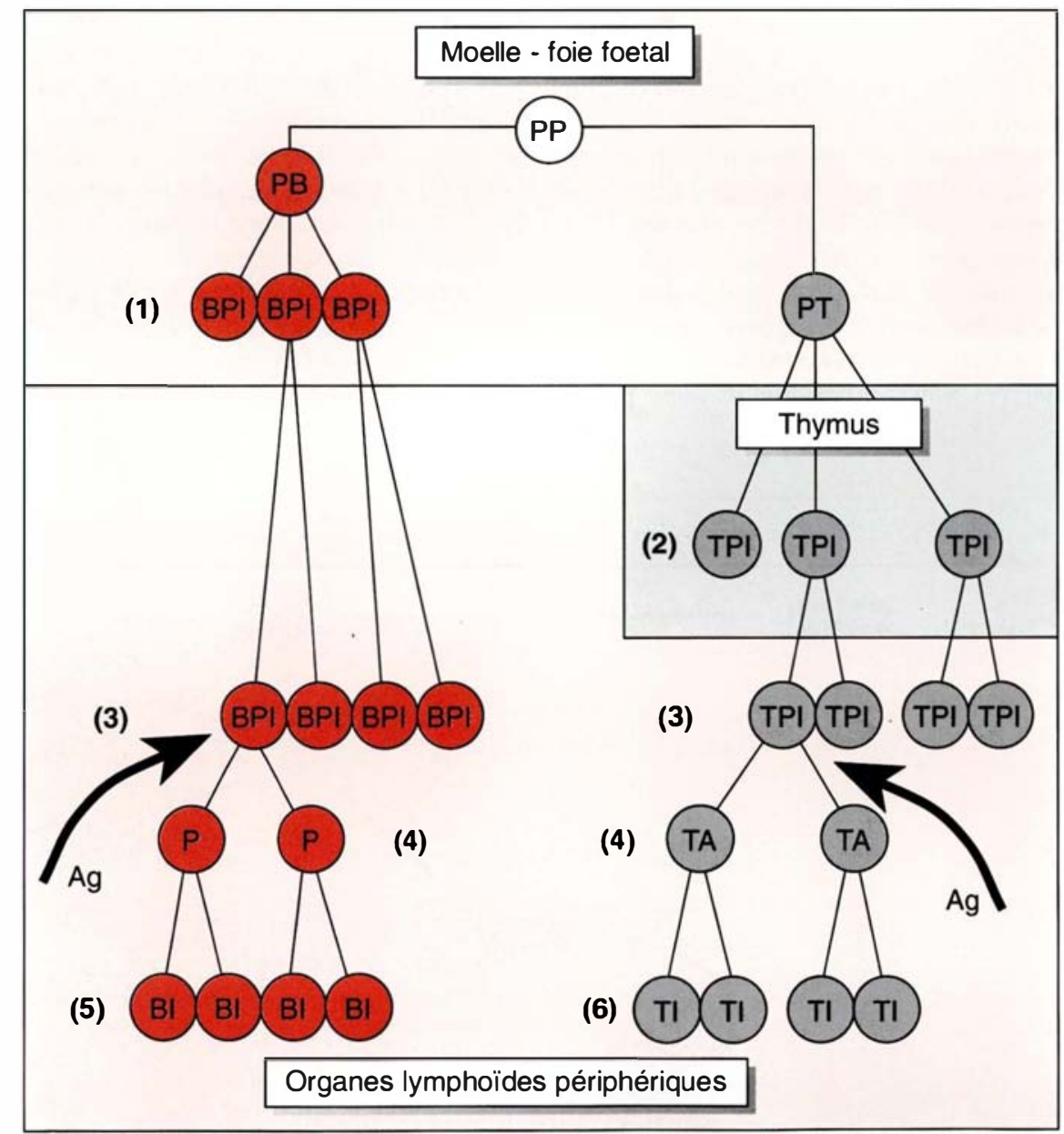

Figure 5. Ontogenèse des lymphocytes $T$ et $B$ après la naissance. (1) Délétion des clones $B$ autoréactifs dans la moelle. (2) Délétion des clones $T$ autoréactifs dans le thymus. (3) Stimulation par l'Ag dans les organes lymphoïdes périphériques après la naissance. (4) Expansion clonale et mise en action permettant l'élimination du pathogène. (5) Développement d'un pool de lymphocytes $B$ immuns produisant des immunoglobulines de divers isotypes et de haute affinité pour l'antigène. (6) Développement d'un pool de lymphocytes $T$ immuns particulièrement efficaces. $P P$ : progéniteur pluripotent; $P B$ : lymphocytes pré-B; $B P I$ : lymphocytes $B$ pré-immuns; $P$ : plasmocytes ; $B I$ : lymphocytes $B$ immuns; $P T$ : prothymocytes; TPI: lymphocytes $T$ préimmuns; TA: lymphocytes $T$ activés; $T I$ : lymphocytes $T$ immuns. 
tes. Les IgG présentes dans le sérum des nouveau-nés sont quasi exclusivement d'origine maternelle. Leur passage transplacentaire s'cffectue au cours du troisième trimestre de la grossesse. Elles assurent ainsi, à la naissance, unc protection partielle de l'enfant contre des infections par des agents bactériens vis-à-vis desquels la mère est elle-même immunisée.

Après la naissance, les stimulations antigéniques itératives vont contribuer à la poursuite de la différenciation et de la maturation des lymphocytes $\mathrm{B}$. L'interaction des IgM membranaires avec les antigènes se fait dans les organes lymphoïdes périphériques. Elle permet la progression des lymphocytes B vers un stade plus avancé de leur différenciation. Cette maturation se traduit par l'cxpression de nouvelles molécules à la surface des lymphocytcs, dont certaines sont des récepteurs pour des interleukines. L'action, sclon une séquence ordonnée, de l'antigène puis de diverses interleukines permet au lymphocyte B de se transformer en plasmocyte et de sécréter des Ig sous forme soluble. La majorité des anticorps secrétés par des lymphocytes B stimulés pour la premic̀re fois par un antigène, sont de nature IgM. Des phénomènes de commutation isotypique sous le contrôlc des intcrleukincs produites par les lymphocytes $\mathrm{T}$ auxiliaires permettent la synthc̀se ultérieure des autres isotypes d'Ig (A, E, G) par ces mêmes lymphocytes B $(\mathrm{m} / \mathrm{s}$, lexique immunologie, suppl. au $n^{\circ} 1$, vol. 5, p. 5). L'accroissement de l'efficacité d'une réponse immunitaire humoralc est alors assuréc par l'expansion préférenticllc des clones $\mathrm{B}$ exprimant des Ig de forte affinité pour l'antigène, clle-même secondaire à des mutations somatiques intervenant au niveau du site de reconnaissance des Ag par les Ig [29, 30]. Les Ig sécrétées sont d'affinité croissante au cours d'une réponse immunitaire, ce qui contribue à améliorer les performances du système à l'égard de l'antigènc en cause. De même que les lymphocytes $\mathrm{T}$, après une primo-invasion, les lymphocytes B garderont la " mémoire " de leur premier contact avec l'antigène. Cette maturation se traduit par une plus grande efficacité fonctionnelle. Elle contribue, conjointement à la persistance d'Ig sériques

$\mathrm{m} / \mathrm{s} n^{\circ} 3$ vol. 9 , mars 93 de forte affinité, à protéger l'individu contre des infections par des microorganismes de même antigénicité.

\section{Conclusion}

L'ensemble des changements quantitatifs et qualitatifs des lymphocytes B et $\mathrm{T}$ induits au cours et au décours des stimulations antigéniques itératives permet la mise en place d'un système de défense humorale et cellulaire efficace. A la naissance, l'immaturité de ce système est responsable en partie de la sévérité des infections néonatales. L'efficacité de thérapcutiques de supplémentation telles que les transfusions d'IgG chez les prématurés a été évaluée depuis dix ans. L'indication de leur utilisation à visée préventive est controversée. La dernière étude publiée [31] conclut à une absence de bénéfice, à des effets secondaires et à un coût économique important. Chez un enfant infecté, la transfusion d'IgG à visée curatrice devrait améliorer le pouvoir opsonisant du sérum et la phagocytose. Une étude très récentc en a évalué l'efficacité sur le pronostic des infections à streptocoques $\mathrm{B}$ à révélation précoce [32]. Les IgG réduisent nettement lc taux de mortalité dans les premiers jours mais ne modifient pas le taux de mortalité globalc.

A côté d'une immaturité de l'immunité spécifique (lymphocytes $\mathrm{B}$ et $\mathrm{T}$ ), il existe également un déficit de l'immunité non spécifique chez le nouveau-né. L'immunité non spécifique pcut être amélioréc par la transfusion de plasma frais qui, outre l'apport d'IgG, restaure le taux dc complément qui est déficitaire. La transfusion de leucocytes a également été proposée, en particulier dans les sepsis avec neutropénic [33]. Toutefois, les risques de complications pulmonaires, de réaction de greffon contre l'hôte et de transmission virale limitent son utilisation.

Des progrès futurs dans la compréhension de mécanismes responsables de l'immaturité de l'immunité spécifique et non spécifique pourraient conduire à proposer de nouvclles thérapeutiques chez le nouveau-né à risque dans l'objectif d'améliorer les performances de son système immunitaire

\section{Summary}

Towards the identification of the molecular basis responsible for the immaturity of the immune system at birth

Early differentiation of T-cell progenitors in the thymus can be followed by analysis of both the expression of various CD cell surface markers and the expression of rearranged genes that encode $\mathrm{TcR}$ and surface Ig receptors. Once they have expressed TcR, autoreactive thymocytes are deleted while thymocytes that can potentially recognize foreign antigens in association with self MHC molecules are positively selected. Most of these processes which result in self tolerance take place during the prenatal life. At birth, the immune system of human newborns is partially immature resulting in deficiency in secretion of lymphokines, cell-mediated cytolysis and Ig synthesis. One of the fundamental differences between adult and newborn immune functions resides in prior exposure to antigen or not. After birth, antigenic challenges are responsible for transient expression of immune functions. They also lead to further differentiation of lymphocytes towards an immunologic memory state. This state improves immune functions and results in protection against further infection by antigenically related pathogens. The identification of the molecular basis responsible for these functional changes is now in progress.

\section{TIRÉS A PART}

G. Sterkers 